\title{
ON AN EIGENVALUE PROBLEM OF AHMAD AND LAZER FOR ORDINARY DIFFERENTIAL EQUATIONS
}

\author{
MARCELLINO GAUDENZI
}

\begin{abstract}
In connection with a problem posed by S. Ahmad and A. C. Lazer, we show the existence of a class of nonselfadjoint eigenvalue problems related to the equation $y^{(n)}+\lambda p(x) y=0$ for which the general eigenvalues comparison is not true. We use a comparison principle for the zeros of the corresponding Cauchy problem.
\end{abstract}

This paper provides a contribution to the understanding of a problem raised by S. Ahmad and A. C. Lazer [1] in connection with the comparison of the eigenvalues for some multi-point boundary value problems which are not selfadjoint.

One is given the equation

$$
L_{n} y+\lambda p(x) y=0
$$

where $p(x)$ is a continuous function of constant sign on an interval $I, \lambda$ is a parameter, and $L_{n} y$ is a linear differential disconjugate operator of order $n$, that is, the only solution of $L_{n} y=0$ with $n$ zeros on $I$ (counting multiplicity) is $y \equiv 0$.

Let us consider the eigenvalue problem given by equation (1) and the system of boundary conditions

$$
\begin{array}{ll}
L_{i} y(a)=0, & i \in\left\{i_{1}, \ldots, i_{k}\right\}, \\
L_{j} y(b)=0, & j \in\left\{j_{1}, \ldots, j_{n-k}\right\},
\end{array}
$$

where $a, b \in I, 1 \leq k \leq n-1, L_{i} y, i=0, \ldots, n-1$, are the quasi-derivatives of $y(x)$ (see $[7]$ ), and $\left\{i_{1}, \ldots, i_{k}\right\},\left\{j_{1}, \ldots, j_{n-k}\right\}$ are two arbitrary sets of indices from the set $\{0, \ldots, n-1\}$.

Problems of this type have been studied extensively (cf. $[2,3,5])$. In particular, Elias [5] has shown that if $(-1)^{n-k} p(x)<0$, then the eigenvalues of problems (1) and (2) are real and nonnegative and form a divergence sequence $\left\{\lambda_{m}\right\}_{m \in N}$.

Ahmad and Lazer [1] have considered a particular type of boundary condition (2), that is

$$
\begin{aligned}
& y(a)=y^{\prime}(a)=\cdots=y^{(k-1)}(a)=0, \\
& y(b)=y^{\prime}(b)=\cdots=y^{(n-k-1)}(b)=0,
\end{aligned}
$$

and showed that if we set $p=p_{i}$, where $p_{i}, i=1,2$, are two continuous functions, considering the corresponding sequence of eigenvalues $\left(\lambda_{i, m}\right)_{m \in \mathbf{N}}, i=1,2$, ordered by magnitude, then the condition

$$
(-1)^{n-k} p_{2}(x) \leq(-1)^{n-k} p_{1}(x)<0
$$

Received by the editors October 4, 1985.

1980 Mathematics Subject Classification (1985 Revision). Primary 34C10.

Key words and phrases. Linear, eigenvalue, comparison, extremal points. 
implies that

$$
\lambda_{1,1} \lambda_{1,2} \cdots \lambda_{1, m} \geq \lambda_{2,1} \lambda_{2,2} \cdots \lambda_{2, m}
$$

for every $m \geq 1$. In the same paper they have raised the question of studying when the condition (4) also implies

$$
\lambda_{1, m} \geq \lambda_{2, m} \quad \text { for every } m \geq 1 ;
$$

an assertion that is true in the selfadjoint case, that is when the operator $L$ is selfadjoint, $n$ is even, and $k=n / 2$.

This paper aims at pointing out a general class of eigenvalue problems (1), (2) for which the eigenvalues' comparison does not follow from condition (4).

In the following we consider the operator $L_{n} y=y^{(n)}$ and the case for which only one condition is set at one of the end points $a$ or $b$, that is $k=1$ or $k=n-1$. Since for $n=2$ the problem is selfadjoint, in the following we also suppose that $n \geq 3$. According to this assumption, the problem (1), (2) becomes

$$
\begin{gathered}
y^{(n)}+\lambda p(x) y=0, \\
y^{\left(i_{1}\right)}(a)=\cdots=y^{\left(i_{k}\right)}(a)=0, \\
y^{\left(j_{1}\right)}(b)=\cdots=y^{\left(j_{n-k}\right)}(b)=0
\end{gathered}
$$

with $k=1$ and $(-1)^{n-1} p(x)<0$ or $k=n-1$ and $p(x)>0$.

We prove the following:

THEOREM 1. Let $p_{1}(x)$ be continuous on $[a, b]$ with $(-1)^{n-k} p_{1}(x)<0$. For every $m \geq 2$ there exist $p_{2}(x) \in C[a, b]$ such that (4) is satisfied but $\lambda_{2, m}>\lambda_{1, m}$.

We obtain this theorem as a consequence of the following result regarding extremal points. The $i$ th extremal point $\theta_{i}(a)(\mathrm{cf}$. [6]), relative to the equation

$$
y^{(n)}+p(x) y=0
$$

and system (6), is defined (when it exists) as the $i$ th value of $b$ in $(a, \infty)$ for which there exists a nontrivial solution of (7) which satisfies (6).

Let us suppose now that $k=n-1$; in agreement with Butler and Erbe [3] we say that the system (6) is admissible if, having called $s$ the unique index from $0, \ldots, n-1$ that does not belong to $\left\{i_{1}, \ldots, i_{n-1}\right\}$, we have $j_{1} \leq s$. If we set $p(x)=p_{j}(x), j=1,2$, in (7), then the corresponding $i$ th extremal point is indicated by $\theta_{j, i}$.

THEOREM 2. Let $p_{1}(x)$ and $m$ be given, where $p_{1}(x)$ is continuous and positive on $[a, \infty)$, and $m \geq 1[m \geq 2]$, and suppose that $\theta_{1, m}$ exists. If system (6) is not admissable $[$ admissible $]$ there exists $p_{2}(x) \in C[a, \infty)$ such that $p_{2}(x) \geq p_{1}(x)>0$, $\theta_{2, m}$ exists, and $\theta_{2, i}>\theta_{1, i}$ for $1 \leq i \leq m[2 \leq i \leq m]$.

We remark that if (6) is admissible, then $\theta_{2,1} \leq \theta_{1,1}$ (see [2, Theorem 2]).

A comparison principle. Let us begin by stating some notation which we use in the following.

We say that a nonnull vector of $\mathbf{R}^{n}, \eta=\left(\eta_{1}, \ldots, \eta_{n}\right)$, has the $D$-property if there exist no three indices $i, j, k$ such that $i<j<k$ and $\eta_{i} \eta_{j}<0 ; \eta_{j} \eta_{k}<0$. 
We say that $\eta$ has the strictly $D$-property if there exists an index $i$ such that the real numbers $\eta_{1}, \ldots, \eta_{i-1},(-1) \eta_{i+1}, \ldots,(-1) \eta_{n}$ are all different from zero and have the same sign.

If $\eta$ has the $D$-property, we denote by $r(\eta)$ the greatest index such that $\eta_{r(\eta)} \neq 0$ and $\eta_{r(\eta)} \eta_{i} \geq 0$ for every $i \leq r(\eta)$.

Now let $y(x)$ be the solution of the Cauchy problem

$$
y^{(n)}+p(x) y=0, \quad y^{(i)}(\xi)=\eta_{i+1}, \quad i=0,1, \ldots, n-1,
$$

with $\xi \in \mathbf{R}$ and $p(x)>0$.

If $\eta_{i}=\delta_{i, l}$ for a given $l, 1 \leq l \leq n$, the solution of (8) will be denoted by $u_{l}(x)$. These solutions will also be called the principal solution of $(8)$.

Every solution $y(x)$ of $(8)$ can have only isolated zeros in a compact interval $[\xi, c], c>\xi$ (cf. [4, Proposition 1, p. 81]). Also, for the form of the equation and Rolle's theorem the quasi-derivatives of $y(x)$, that is $y(x), y^{\prime}(x), \ldots, y^{(n-1)}(x)$, can have only isolated zeros.

Let $z_{1}<\cdots<z_{m}$ be the ordered set of the zeros (eventually empty) of the quasiderivatives of $y(x)$ in an interval $(\xi, c]$ and let $Y(x)$ be the vector $\left(y(x), y^{\prime}(x), \ldots\right.$, $\left.y^{(n-1)}(x)\right)$.

LEMMA 1. If $\eta$ has the D-property, then $Y(x)$ has the strictly D-property for $x>\xi$. Moreover $y^{(j)}(x), 0 \leq j \leq n-1$, vanishes at the point $z_{i}, i \geq 1$, if and only if $j \equiv(r(\eta)-i) \bmod n$.

PROOF. It is not restrictive to assume $\eta_{r(\eta)}>0$. Let $\varepsilon>0$ such that $0<\varepsilon<$ $z_{1}-\xi$. In the interval $(\xi, \xi+\varepsilon)$ the functions $y^{(i)}(x)$ are all positive and increasing for $i=0, \ldots, r(\eta)-2$; all negative and decreasing for $i=r(\eta), r(\eta)+1, \ldots, n-1$, while $y^{(r(\eta)-1)}(x)$ is positive and decreasing. This situation can change only if $y^{(r(\eta)-1)}(x)$ vanishes. Therefore, if $z_{1}$ exists, it must be a zero of $y^{(r(\eta)-1)}(x)$, moreover only this quasi-derivative of $y(x)$ vanishes at this point, and $Y(x)$ has the strictly $D$-property for $x \in\left(\xi, z_{1}\right]$. This argument can be repeated in every interval $\left(z_{i}, z_{i+1}\right], i=1, \ldots, m-1$, proving the lemma.

Let $j, 0 \leq j \leq n-1$, be a fixed index and consider the functions $u_{1}^{(j)}(x), u_{2}^{(j)}(x)$, $\ldots, u_{n}^{(j)}(x)$. Denote by $w_{1}<\cdots<w_{m}$ the ordered set (possibly empty) of the zeros of these functions on an interval $(\xi, c]$.

LEMMA 2. $u_{l}^{(j)}(x), 1 \leq l \leq n$, vanishes at the point $w_{i}, i \geq 1$, if and only if $l \equiv(i+j) \bmod n$.

PROOF. The functions $u_{l_{1}}^{(j)}(x), u_{l_{2}}^{(j)}(x), l_{1} \neq l_{2}$, cannot have a common zero $w_{i}$ on $(\xi, c]$, otherwise there is a nontrivial linear combination $v(x)$ of them with two quasi-derivatives which vanish at $w_{i}$; since the vector $\left(v(\xi), v^{\prime}(\xi), \ldots, v^{(n-1)}(\xi)\right)$ has the $D$-property, this is in contradiction to Lemma 1. Moreover between two zeros of $u_{l_{1}}^{(j)}(x)$ there is a zero of every function $u_{l}^{(j)}(x), l \neq l_{1}$; otherwise there exists (see [4, Lemma 1, p. 4]) a nontrivial linear combination of two principal solutions with two quasi-derivatives which vanish at a point $x_{0}>\xi$, again in contradiction to Lemma 1.

Since $u_{j+1}^{(j)}(\xi)=1$ and $u_{l}^{(j)}(\xi)=0$ for every $l \neq j+1$, from the preceding observations it follows that if $w_{1}$ exists, it must be a zero of $u_{j+1}^{(j)}(x)$. 
Suppose now that the lemma is true for $w_{1}, \ldots, w_{i}$, but not for $w_{i+1}$. This means that $w_{i}$ is a zero of $u_{l}^{(j)}(x)$ and, if $l<n[l=n]$, that $w_{i+1}$ is a zero of $u_{l_{1}}^{(j)}(x)$, with $l_{1} \neq l+1\left[l_{1} \neq 1\right]$. Since all zeros $w_{t}, t \leq i$, are simple, it follows that

$$
u_{l}^{(j)}\left(w_{i+1}\right) u_{l+1}^{(j)}\left(w_{i+1}\right)<0 \quad\left[u_{n}^{(j)}\left(w_{i+1}\right) u_{1}^{(j)}\left(w_{i+1}\right)>0\right] .
$$

So there exists $\alpha>0[\alpha<0]$ for which $v_{1}(x)=u_{l}(x)+\alpha u_{l+1}(x)\left[v_{1}(x)=u_{n}(x)+\right.$ $\left.\alpha u_{1}(x)\right]$ is such that $v_{1}^{(j)}\left(w_{i+1}\right)=0$. As $u_{l_{1}}^{(j)}\left(w_{i+1}\right)=0$, there exists a nontrivial linear combination $v_{2}(x)$ of $v_{1}(x)$ and $u_{l_{1}}(x)$ which has two quasi-derivatives which vanish at $w_{i+1}$, but the vector $\left(v_{2}(\xi), \ldots, v_{2}^{(n-1)}(\xi)\right)$ has the $D$-property and this contradicts Lemma 1.

The following proposition gives us a criterion to compare the zeros of two solutions of the Cauchy problem (8).

Proposition. Suppose that $u_{l}^{(j)}(x), j+1 \leq l$, has $m$ zeros, $w_{1}<\cdots<w_{m}$, on $(\xi, c]$. If $\eta$ is a vector with the D-property such that $j+1 \leq r(\eta) \leq l$ and $\eta_{i} \neq 0$ for at least one index $i \neq l$, then the $j$-derivative of the solution $y(x)$ of (8) has at least $m$ zeros $z_{1}<\cdots<z_{m}$ on $\left(\xi, w_{m}\right)$ and $z_{i}<w_{i}$ for every $i$. Moreover if $l=r(\eta), y^{(j)}(x)$ has exactly $m$ zeros on $\left(\xi, w_{m}\right)$.

PROOF. It is not restrictive to assume $\eta_{r(\eta)}>0$, so that $\eta_{i} \geq 0$ for $1 \leq i \leq r(\eta)$, $\eta_{i} \leq 0$ for $r(\eta)+1 \leq i \leq n$. Suppose first that $l=r(\eta)$. From Lemma 2 it follows that at the point $w_{i}$ we have for all the indices $t \neq l$, either $\eta_{t}=0$ or $\operatorname{sgn}\left[\eta_{t} u_{t}^{(j)}\left(w_{i}\right)\right]=(-1)^{i}$. Since $\eta_{t} \neq 0$ for at least an index $t \neq l$, from the relation $y^{(j)}(x)=\sum_{i=1}^{n} \eta_{i} u_{i}^{(j)}(x)$ and by continuity it follows that $y^{(j)}(x)$ has a zero in every interval $\left(w_{i}, w_{i+1}\right), i=1, \ldots, m-1$. But $r(\eta) \geq j+1$ so that $y^{(j)}(x)>0$ for $\xi<x<\xi+\varepsilon$ and $\varepsilon$ sufficiently small; this implies that $y^{(j)}(x)$ must have a zero also in the interval $\left(\xi, w_{1}\right)$. If $y^{(j)}(x)$ has two zeros in an interval $\left(w_{i}, w_{i+1}\right)$ or $\left(\xi, w_{1}\right)$, then it is possible to consider a linear combination $v(x)$ of $y(x)$ and $u_{l}(x)$ which has two quasi-derivatives which vanish at a point $x_{0}>\xi$. Since $r(\eta)=l$, the initial conditions of $v(x)$ determine a vector with the $D$-property and this contradicts Lemma 1.

If $l>r(\eta)$, then by Lemma $2 u_{r(\eta)}^{(j)}(x)$ has $m$ zeros $w_{1}^{\prime}<w_{2}^{\prime}<\cdots<w_{m}^{\prime}$ on $\left(\xi, w_{m}\right)$ and $w_{i}^{\prime}<w_{i}$ for every $i$. Now if $\eta_{i} \neq 0$ only for $i=r(\eta)$, then the proof is trivial; otherwise the conclusion follows from the case $l=r(\eta)$.

We consider now the particular case of problem (8) for which $\xi=0$ and $p(x)$ is constant, that is $p(x)=k^{n}, k>0$. The problem becomes

$$
y^{(n)}+k^{n} y=0, \quad y^{(i)}(0)=\eta_{i+1}, \quad i=0,1, \ldots, n-1 .
$$

Since in this case we are interested in the dependence of $k$, we indicate the solution of (9) with $y(x, k)$ and the principal solutions with $u_{l}(x, k), 1 \leq l \leq n$.

For every $k>0$ the principal solutions are oscillatory (see [6, Remark, p. 188]). If $\eta$ is a vector with the $D$-property, then from the Proposition the solution of (9) is also oscillatory for every $k$. Then it is possible to consider the function $h(k)$ which associates the abscissa of the first zero of $y(x, k)$ in the interval $(0,+\infty)$ to $k$.

LEMMA 3. Let $\eta$ be a vector with the D-property and $y(x, k)$ be the solution of (9). Then

$$
\lim _{k \rightarrow+\infty} k h(k)=M>0
$$


and

$$
\lim _{k \rightarrow+\infty} \frac{\partial^{(i)} y}{\partial x^{(i)}}(h(k), k) / \frac{\partial^{(i-1)} y}{\partial x^{(i-1)}}(h(k), k)=+\infty
$$

for every $i$ such that $2 \leq i \leq n-1$.

ProOF. From the relations

$$
\begin{aligned}
& y(x, k)=\sum_{i=1}^{n} \eta_{i} u_{i}(x, k), \\
& u_{i}(x, k)=k^{1-i} u_{i}(k x, 1), \quad i=1,2, \ldots, n,
\end{aligned}
$$

if follows that

$$
\frac{\partial^{(j)} y}{\partial x^{(j)}}(h(k), k)=\sum_{i=1}^{n} \eta_{i} k^{1-i+j} \frac{\partial^{(j)} u_{i}}{\partial x^{(j)}}(k h(k), 1) .
$$

For our definition, $h(k)$ is the first positive zero $y(x, k)$, therefore $k h(k)$ is the first positive zero of $y(x / k, k)=\sum_{i=1}^{n} \eta_{i} k^{1-i} u_{i}(x, 1)$. Let $t$ be the first index such that $\eta_{t} \neq 0$. For $k \rightarrow+\infty, k h(k)$ tends to the first positive zero $w_{1}$ of $u_{t}(x, 1)$. Since $\left(\partial^{(j)} u_{t} / \partial x^{(j)}\right)\left(w_{1}, 1\right) \neq 0$ for $j=1,2, \ldots, n-1$ by Lemma 1 , the proof of the lemma then follows by relation (11).

Proof of Theorem 2. Let system (6) be nonadmissible.

Let $s$ be the unique index which does not belong to $\left\{i_{1}, i_{2}, \ldots, i_{n-1}\right\}$; then $\theta_{l, i}(a)$, $l=1,2$, is the $i$ th zero of the $j_{1}$ th derivative of the solution $u_{s+1}(x)$ of $(8)$, where $p(x)=p_{l}(x)$ and $\xi=a$. Let $x_{1}$ be the first zero greater than $a$ of $u_{s+1}(x)$. Since $j_{1}>s$, from Lemma 1 it follows that $a<x_{1}<\theta_{1,1}(a)$. We denote also by $u_{*}(x)$ the principal solution $u_{n}(x)$ of $(8)$, where $p(x)=p_{1}(x)$ and $\xi=x_{1}$, and by $\theta_{i}\left(x_{1}\right)$ the $i$ th zero greater than $x_{1}$ of $u_{*}^{\left(j_{1}\right)}(x)$.

Let us suppose first that $\theta_{m}\left(x_{1}\right)$ exists. By Lemma $1, u_{s+1}^{(i)}\left(x_{1}\right)<0$ for $i=$ $1, \ldots, n-1$. Applying the Proposition with $\xi=x_{1}$ and $l=n$ it results that $\theta_{i}\left(x_{1}\right)>$ $\theta_{1, i}(a)$ for $i=1, \ldots, m$. Since the zeros $\theta_{i}\left(x_{1}\right)$ are simple, by the continuous dependence of the initial conditions and the Proposition there exists $\bar{x}<x_{1}$ and $\delta>0$ such that for every vector $\gamma, \gamma=\left(\gamma_{1}, \gamma_{2}, \ldots, \gamma_{n}\right)$, with $\gamma_{n}=1$ and $0 \leq \gamma_{i} \leq \delta$ for $i=1, \ldots, n-1$, and for every $x_{0} \in\left[\bar{x}, x_{1}\right]$ the $j_{1}$ th derivative of the solution of the problem

$$
y^{(n)}+p_{1}(x) y=0, \quad y^{(i)}\left(x_{0}\right)=\gamma_{i+1}, \quad i=0, \ldots, n-1,
$$

has exactly $m$ zeros $z_{1}<\cdots<z_{m}$ in $\left(x_{0}, \theta_{m}\left(x_{1}\right)\right)$ and we have that

$$
\theta_{1, i}(a)<z_{i}, \quad i=1, \ldots, m \text {. }
$$

Now let $\eta$ be the vector whose components are $\eta_{i}=u_{s+1}^{(i-1)}(\bar{x})$. By Lemma 3 , there exists $k_{0}$ such that $h\left(k_{0}\right)+\bar{x}<x_{1}, k_{0}^{n}>\max \left\{p_{1}(x), x \in\left[a, x_{1}\right]\right\}$, and if $y(x, k)$ is the solution of (9) it follows that

$$
0<\frac{\partial^{(i)} y}{\partial x^{(i)}}\left(h\left(k_{0}\right), k_{0}\right) / \frac{\partial^{(n-1)} y}{\partial x^{(n-1)}}\left(h\left(k_{0}\right), k_{0}\right)<\delta, \quad i=1, \ldots, n-2
$$


Consider the function

$$
\tilde{p}(x)= \begin{cases}p_{1}(x) & \text { for } a \leq x \leq \bar{x} \\ k_{0}^{n} & \text { for } \bar{x}<x \leq \bar{x}+h\left(k_{0}\right), \\ p_{1}(x) & \text { for } x>\bar{x}+h\left(k_{0}\right) .\end{cases}
$$

For Lemma 1 the $j_{1}$ th derivative of the solution $\tilde{u}_{s+1}(x)$ of $(8)$ with $p(x)=\tilde{p}(x)$ and $\xi=a$ does not vanish in $\left(a, \bar{x}+h\left(k_{0}\right)\right]$; from (13) and (12) it follows then that the $i$ th zero of $\tilde{u}_{s+1}^{(j)}(x)$ is greater than $\theta_{i, 1}(a)$ for every $i \leq m$. The existence of a continuous function $p_{2}(x) \geq \tilde{p}(x)$ which verifies the theorem then follows by the fact that the zeros of $\tilde{u}_{s+1}^{(j)}(x)$ are simple and from the classical result on differential equations.

Consider now the case for which $\theta_{m}\left(x_{1}\right)$ does not exist. Since the principal solutions of (9) are oscillatory, from (10) and Rolle's theorem it follows that the $i$ th, $i \geq 1$, zero of $u_{s+1}^{\left(j_{1}\right)}(x, k)$ tend to zero for $k \rightarrow+\infty$. By Lemma 1 the vector $\eta$, whose components are $\eta_{i}=u_{s+1}^{(i-1)}\left(\theta_{1, m}(a)\right), i=1, \ldots, n$, has the $D$-property, therefore for the Proposition also the $i$ th zero of the $j_{1}$ th derivative of the solution of (9) which correspond to this vector tends to zero for $k \rightarrow+\infty$. So it is possible to consider a function $p_{1}^{\prime}(x)$ such that $p_{1}^{\prime}(x) \geq p_{1}(x), p_{1}^{\prime}(x)=p_{1}(x)$ for $a \leq x \leq \theta_{1, m}(a)$, and the point $\theta_{m}\left(x_{1}\right)$ corresponding to the new function $p_{1}^{\prime}(x)$ exists. The proof of the theorem then follows from the preceding case.

Let (6) be admissible.

By Lemma 1 the first zero $x_{1}$ of $u_{s+1}(x)$ belongs to the interval $\left[\theta_{1,1}(a), \theta_{1,2}(a)\right)$. Therefore if we proceed in the same way as in the case for which system (6) is not admissible, we can prove the existence of a function $p_{2}(x) \geq p_{1}(x)$ such that $\theta_{2, i}(a)>\theta_{1, i}(a)$ for $2 \leq i \leq m$ and this completes the proof of the theorem.

Proof of Theorem 1. Suppose first that $k=n-1$.

The function $p_{1}(x)$ can be considered to be defined on all of the interval $[a,+\infty)$ setting $p_{1}(x)=p_{1}(b)$ for $x>b$. If system (6) is admissible, then $\lambda_{1,1}>0$ (see [5, Corollary 3]). Moreover $\lambda_{1, m}$ is the $m$ th eigenvalue of problem (5), (6), where $p(x)=p_{1}(x)$, if and only if $b$ is the $m$ th extremal point relative to equation $y^{(n)}+\lambda_{1, m} p_{1}(x) y=0$ and system (6) (see [5, Theorem 3]). By Theorem 2 there exists $p_{2}(x) \geq p_{1}(x)$ such that the $m$ th $(m \geq 2)$ extremal point relative to the equation $y^{(n)}+\lambda_{1, m} p_{2}(x) y=0$ and system (6) is greater than $b$. Since the positive eigenvalues of $(1),(2)$ are decreasing functions of the point $b$ (see [6, Corollary 5]), the $m$ th eigenvalue $\lambda_{m}$ of problem (5), (6), where $p(x)=\lambda_{1, m} p_{2}(x)$, is greater than 1. Therefore $\lambda_{m}=\lambda_{2, m} / \lambda_{1, m}>1$ and then $\lambda_{2, m}>\lambda_{1, m}$.

If the system (6) is not admissible, then $\lambda_{1,1}=0$ and $\lambda_{1, m}>0$ for $m \geq 2$; therefore we can prove the theorem as in the preceding case using Theorem 2 .

Suppose now that $k=1$.

We remark that $y(x)$ is a solution of problem (5), (6) if and only if the function $z(x)=y(b+a-x)$ is a solution of problem

$$
\begin{gathered}
z^{(n)}+(-1)^{n} \lambda p(b+a-x) z=0 \\
z^{\left(j_{1}\right)}(a)=\cdots=z^{\left(j_{n-k}\right)}(a)=0 \\
z^{\left(i_{1}\right)}(b)=\cdots=z^{\left(i_{k}\right)}(b)=0 .
\end{gathered}
$$


Therefore the eigenvalues of problem (5), (6) are the same as the eigenvalues of problem (14), (15). It follows that the case $k=1$ can be reduced to the case $k=n-1$, and this completes the proof of the theorem.

ACKNOWLedgment. I am grateful to Professor Giovanni Vidossich for suggesting this research and to Professor A. C. Lazer for interesting discussion.

\section{REFERENCES}

1. S. Ahmad and A. C. Lazer, On n-th order Sturmian theorey, J. Differential Equations 35 (1980), 87-112.

2. $\ldots$, On an extension of the Sturm comparison theorem, SIAM J. Math. Anal. 12 (1981), 1-9.

3. G. J. Butler and L. H. Erbe, Integral comparison theorems and extremal points for linear differential equations, J. Differential Equations 47 (1983), 214-226.

4. W. A. Coppel, Disconjugacy, Lecture Notes in Math., vol. 20, Springer-Verlag, New York, 1955.

5. U. Elias, Eigenvalue problem for the equations $L y+\lambda p(x) y=0$, J. Differential Equations 29 (1978), 28-57.

6. __ Oscillatory solutions and extremal points for a linear differential equation, Arch. Rational Mech. Anal. 76 (1979), 177-198.

7. $\ldots$ The extremal solutions of the equations $L y+p(x) y=0$. II, J. Math. Anal. Appl. 55 (1976), 253-265.

INTERNational School For AdVANCEd Studies, Strada Costiera 11, 34014 TriESTE, ITALY Italy

Current address: Istituto di Matematica, Informatica e Sistemistica Via Zanon, 6, 33100 Udine, 